\title{
COMPUTATIONAL STUDY OF THE NON-PARAMETRIC SQUEEZE FILM DAMPER BEARING INVERSE MODEL BASED ON ARTIFICIAL NEURAL NETWORKS APPLIED TO A ROTOR-CASING SYSTEM RUNNING ON UNSUPPORTED SFDS
}

DOI:

10.1115/GT2019-90390

\section{Document Version}

Accepted author manuscript

Link to publication record in Manchester Research Explorer

Citation for published version (APA):

Torres Cedillo, S., Al-Ghazal, G., Bonello, P., \& Cortés Pérez, J. (2019). COMPUTATIONAL STUDY OF THE NON-PARAMETRIC SQUEEZE FILM DAMPER BEARING INVERSE MODEL BASED ON ARTIFICIAL NEURAL NETWORKS APPLIED TO A ROTOR-CASING SYSTEM RUNNING ON UNSUPPORTED SFDS. In Proceedings of ASME Turbo Expo 2019: Turbomachinery Technical Conference and Exposition GT2019 June 17-21, 2019, Phoenix, Arizona, USA https://doi.org/10.1115/GT2019-90390

\section{Published in:}

Proceedings of ASME Turbo Expo 2019: Turbomachinery Technical Conference and Exposition GT2019 June 1721, 2019, Phoenix, Arizona, USA

\section{Citing this paper}

Please note that where the full-text provided on Manchester Research Explorer is the Author Accepted Manuscript or Proof version this may differ from the final Published version. If citing, it is advised that you check and use the publisher's definitive version.

\section{General rights}

Copyright and moral rights for the publications made accessible in the Research Explorer are retained by the authors and/or other copyright owners and it is a condition of accessing publications that users recognise and abide by the legal requirements associated with these rights.

\section{Takedown policy}

If you believe that this document breaches copyright please refer to the University of Manchester's Takedown

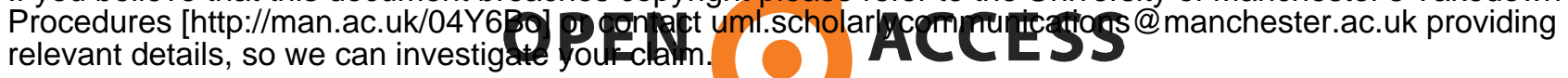


June 17-21, 2019, Phoenix, Arizona USA

GT2019- 90390

\section{COMPUTATIONAL STUDY OF THE NON-PARAMETRIC SQUEEZE FILM DAMPER BEARING INVERSE MODEL BASED ON ARTIFICIAL NEURAL NETWORKS APPLIED TO A ROTOR-CASING SYSTEM RUNNING ON UNSUPPORTED SFDS}

\author{
Sergio G. Torres Cedillo \\ Centro Tecnológico Aragón, FES Aragón, Universidad \\ Nacional Autónoma de México (UNAM), Av. Rancho Seco \\ s/n, Edo. de México, México. C.P. 57130. \\ Philip Bonello \\ School of Mechanical, Aerospace and Civil Engineering, \\ University of Manchester, Manchester, UK
}

\author{
Ghaith Ghanim Al-Ghazal \\ School of Mechanical, Aerospace and Civil Engineering, \\ University of Manchester, Manchester, UK \\ Jacinto Cortés Pérez \\ Centro Tecnológico Aragón, FES Aragón, Universidad \\ Nacional Autónoma de México (UNAM), Av. Rancho Seco \\ s/n, Edo. de México, México. C.P. 57130.
}

\section{Alberto Reyes Solís \\ Centro Tecnológico Aragón, FES Aragón, Universidad Nacional Autónoma de México (UNAM), Av. Rancho Seco s/n, Edo. de México, México. C.P. 57130.}

\begin{abstract}
Squeeze Film Damper (SFD) bearings play a vital role in attenuating large amplitudes of vibration due to their relatively simple assembly in aero engine designs. The modern aeroengine structures, typically, have at least two nested rotors mounted within a flexible casing via squeeze-film damper (SFD) bearings. There is a growing body of research into identification techniques for bearing models for use in rotorbearing analysis to improve reliability and/or efficiency of implementation. The authors' previous work has shown that, for cases where there is no adequate linear connection between the rotor and casing, the identification of the unbalance from externally mounted sensors requires a virtual instrument that can determine the vibration of the rotor relative to the casing, as a substitute for internal instrumentation. The present study is devoted to determining the effectiveness of the inverse SFD model (under different unbalance state conditions), when it is applied to a rotor-casing system, wherein the rotor runs on two unsprung SFD bearings. The validation of the inverse SFD model enables its use in a future study of the identification of unbalance in such complex systems.
\end{abstract}

\section{INTRODUCTION}

Since the publication of a technical study by Cooper [1], a large and growing body of literature has investigated how to model the dynamic behaviour of Squeeze Film Damper (SFD) bearings. SFDs are typically employed on aero-engine assemblies to attenuate vibration and the transmitted forces. An SFD bearing commonly consists of a thin-film of oil pumped into the annular clearance between the outer race of a rolling-element bearing and its housing. The SFD journal (i.e. the outer ring of the rolling-element bearing) is prevented from spinning but is free to orbit within the oil-filled clearance. There are two common designs of SFD assemblies, one being referred to as the "sprung SFD", which has a retainer spring, usually in the form of a squirrel cage, placed in parallel with the damper journal. This spring might be inserted to tune the system's natural frequencies. The primary function of this spring is to support the static load on the journal (normally the gravity load). It also serves to prevent the journal from rotating (instead of dog or anti-rotation pins). It is now well established from a variety of studies [2-4] that it is possible to centre the journal within the annulus of oil by preloading the retainer spring, thereby reducing nonlinearities.

However, to achieve a centred journal in practice is a complex procedure, and the preloading mechanisms might introduce non-desirable problems at the design stage. Moreover, the squirrel cage necessitates axial room and regular maintenance for fatigue issues. Due to this constraint, a typical engine of a leading European aero-engine designer incorporates only one sprung SFD per rotor; the remaining SFDs being unsprung [5]. In this "unsprung" or "unsupported" SFD design, the journal is prevented from rotating by antirotation pins or dogs but is free to orbit within the radial (annular) clearance between the journal and the housing. In the static condition (non-rotating), the unsprung SFD cannot support static loads and it is fully eccentric when the system is not spinning (journal rests at the bottom of the clearance). Hence, this assembly relies on the vibration originating from 
the unbalance forces to generate pressure that supports the static loads at the journal locations.

Holmes and Dogan [6] illustrated the benefits of an SFD in attenuating vibration in critical-speed regions. A great challenge in the analysis of such as rotor-bearing system is to consider the vibration-dependent nonlinear forces from the SFD bearings, which can introduce undesirable phenomena into the structural response that can be aggravated by increasing unbalance. As already stated in [7], the system identification of a nonlinear bearing involves the extraction of its input/output relationship with the component in isolation. The extracted model can then be used within the dynamic analysis of any system in which the identified component is fitted. Identification can be either parametric or nonparametric. The latter is a black box technique that does not involve making any assumptions on the nature of the input/output relationship and it is therefore suited to SFD identification.

Considerable research has focused on the system identification of a forward nonlinear bearing model that outputs the bearing force from a given relative displacement/velocity of the journal relative to the housing as input [8-11]. These models have been utilised in the prediction of the dynamic response from known unbalance forces (i.e. the forward problem). Such a problem relies on non-parametric identification based on the Chebyshev polynomial technique[2-5], which is preferred to advanced numerical models of SFDs.

A pioneer study [12] employed a forward SFD model identified from experimental data using a neural network, which gives more accurate predictions when implemented in the forward problem, since it captures the bearing features that are difficult to model theoretically, such as lack of roundness of the annular clearance profile. Whilst a neural network has been used in the context of rotating machinery unbalance, such as for condition monitoring applications [12], it can also be used as a classifier of unbalance severity and looseness by extracting statistical features from the vibration signals and then, feeding them into network for training $[13,14]$. Also, in [15], the neural network is used to localise unbalance in a particular machine.

There is a relatively small body of literature concerned with the inverse method applied to a rotor-bearing problem. The purpose of this is to identify unbalance using only externally mounted sensors when the source of vibration (i.e. rotor) cannot be accessed under operating conditions [5,7,1618]. In some applications of this kind, the recurrent neural network (RNN) can be used as a substitute for internal instrumentation for use in non-intrusive unbalance identification schemes. As reported in [7], a recurrent neural network was used to identify a non-parametric empirical inverse model of an SFD bearing installed on a simple nonrotating experimental rig. The identified inverse bearing model was then employed in the application of the inverse rotor-bearing problem to the aforementioned non-rotating experimental rig, where the rotor excitation force was identified using only a casing-mounted sensor.
In [5], the relative vibrations at the sprung and unsprung SFD bearings were determined using identified inverse models for each case. These SFD inverse models where based on RNNs and trained to reproduce the Cartesian displacements of the journal relative to the bearing housing, when presented with given input time histories of the Cartesians SFD bearing forces. The inverse method presented in [5] is suitable for balancing generic SFD rotor-dynamic systems that pose a quasi-implicit inverse problem (i.e. with only one weak linear rotor-casing connection, apart from the non-linear ones).

The contribution of this paper is the identification of an inverse SFD model for application to a rotor-casing system wherein the rotor runs only on unsprung SFD bearings. Such a configuration has not been considered in the authors' preceding work [5], which considered only one unsprung SFD. The analysis involves discussions about the ability of the trained network to reliably predict the SFD relative displacement time series, when presented with given input SFD force signals that were measured from the casing under distinct unbalance state conditions that were significantly different from those used in the training stage. Such tests prove that the network has learned the input-output SFD relationship and is therefore able to predict SFD vibration under different system excitation conditions, rather than simply reproducing the training data set information.

Section 2 details the distinct rotordynamic system considered in this paper. In section 3, the forward problem and the generation of SFD forces from external vibrations are described. Section 4 presents the RNN inverse models of the SFD bearings, while section 5 contains discussion of the study cases along with the results evaluation.

\section{FLEXIBLE ROTOR/CASING UNSPRUNG SFD SYSTEM}

This case study is aimed at testing an SFD inverse model based on recurrent neural networks, when a high-pressure rotor (HP) is running on two unsupported SFDs. As already reported in previous authors' works [5,7,19], these inverse models are employed as virtual internal instrumentation to be applied to an inverse unbalance identification method. A low pressure rotor (LP) is not included, since it would be relatively easily to balance by traditional balancing methods as stated by Dicken et al. and Ramlau et al. [16,20]. In contrast to preceding computational works [5,18], the HP rotor has no linear (structural) connections between the rotor and casing, as illustrated in Figure 1.

It can be seen from Figure 1, that the HP rotor is supported on the casing by the two unsupported SFD bearings (left- and right-hand sides). It can also be observed that the only connections between the rotor and casing are the SFDs (oil films) and thus, the casing vibration is related implicitly to the unbalance on the rotor (rather than explicitly by receptance frequency response functions, when the rotor and casing have a structural connection).

The oil film forces that excite the casing are driven by the synchronous response due to unbalance on the rotor and hence, the implicit relation. It is important to emphasise that 
the current SFD system was not considered in $[5,17,18]$. It also should be noted that, as is customary, e.g. in [3,16-18,2124], the rolling element bearing that is surrounded by the SFD (oil film) is regarded as rigid. As in $[5,17,18]$, for the current case study, the elastic support S2 of the casing is taken as being anisotropic $(300 \mathrm{MN} / \mathrm{m}$ and $600 \mathrm{MN} / \mathrm{m}$ in the horizontal and vertical planes, respectively).

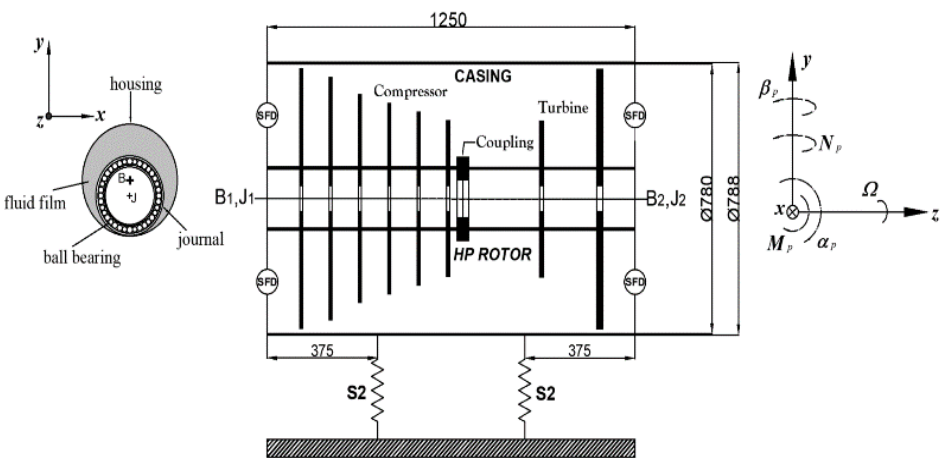

Figure 1. Flexible rotor/casing SFD system (dim in mm).

\subsection{MODAL EIGEN-PARAMETERS}

In $[5,17,18]$, 'forward' and 'inverse' solution methods employed the eigenforms and eigenfrequencies of the linear part of the system (see Fig. 2) under non-rotating conditions, computed from a one-off eigenvalue analysis. The nonlinear rotating system can be regarded as the 'linear part' acted on by the unbalance, static loading and the motion-dependent excitations (nonlinear SFD forces and the gyroscopic effect). As in previous works $[17,18,23,24]$, the 'linear part' of the system is defined as the given system (Figure 1) minus the oil films (replaced by gaps). Therefore, unlike previous work [5], the linear part of the current system is composed of two uncoupled systems: the "free-free" rotor; the casing on its mountings (S2 in Figure 1).

As already reported in [23], SFDs are usually assumed to be the only significant source of damping in aero-engines. Consequently, the linear part can be assumed as undamped, although the following method can easily accommodate proportional damping in the casing structure at no computational cost. In both the horizontal and vertical planes the modal parameters were computed using the finite element model by NASTRAN [25], where the rotor and casing were modelled by beam element and the compressor/turbine disks and couplings ('disks') by concentrated inertia elements.

Based on [5,16-18], the material and geometric properties were taken to enable the calculations of their modal properties. In Fig. 2(a, b), the first seven mode shapes with the eigenfrequencies of the linear part are presented for the flexible rotor/casing SFD system in horizontal and vertical plane, respectively. In each of Figures 2(a), 2(b) the rotor deformation is shown in the upper diagrams and the casing centreline displacement is shown in the lower diagrams. It can be observed in this system that in each plane $x z, y z$, the first two rotor modes will be $0 \mathrm{~Hz}$ modes defining rigid body translation and rotation (about the centre of mass). The highest eigenfrequency considered is $619 \mathrm{~Hz}$. This means that for a top speed of $\sim 200 \mathrm{rev} / \mathrm{s}$, the simulated nonlinear response (with the SFDs in place) can accurately include up to three harmonics. Frequency analysis did not reveal higher harmonics of any significance.
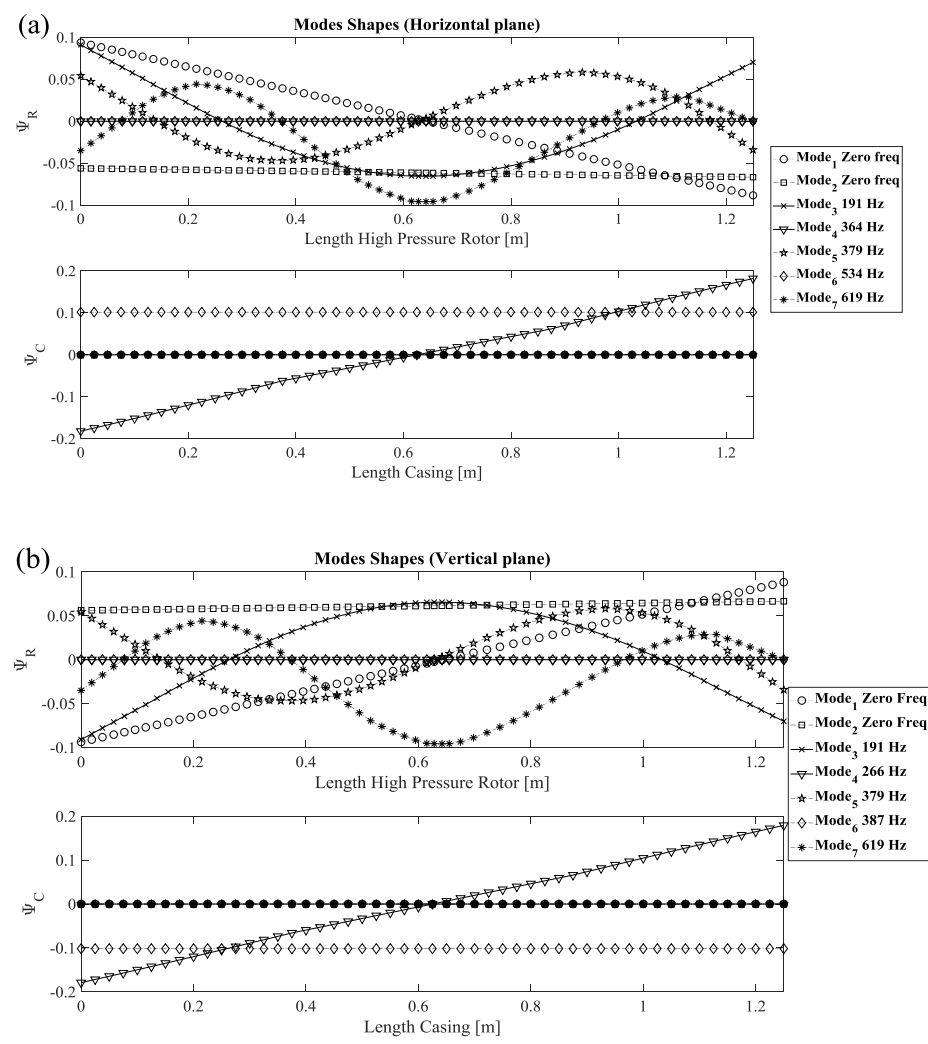

Figure 2. The rotor/casing SFD system eigenforms and eigenfrequencies: (a) horizontal plane (rotor - upper diagram; casing - lower diagram); (b) vertical plane (rotor - upper diagram; casing - lower diagram)

\section{THEORY}

\subsection{FORWARD PROBLEM METHOD}

The forward problem in this research was solved to generate a vibration response for the following two purposes: (1) to generate the required training data to obtain the two SFD inverse models; (2) to investigate and validate the obtained inverse SFD models by using vibration casing data. The data in step (1) are generated by using a chirp signal excitation at two prescribed locations over the rotor, this step being described in detail in Section 4.1. The data in step (2) are generated by applying four different unbalance distributions, being employed to test two SFD non-parametric inverse models. The forward problem is defined in the time domain by the modal equation of motion of the flexible rotor-casing SFD system, which are integrated for $\mathbf{q}$ and $\dot{\mathbf{q}}$, given known excitation $\mathbf{f}_{\mathbf{u}}$ :

$\ddot{\mathbf{q}}+\Lambda \mathbf{q}=\sum_{n=1}^{2} \mathbf{H}_{\mathbf{v}_{n}}{ }^{\mathrm{T}} \mathbf{f}_{\mathbf{v}_{n}}\left(\mathbf{v}_{n}, \dot{\mathbf{v}}_{n}\right)+\mathbf{H}_{\mathbf{u}}{ }^{\mathrm{T}} \mathbf{f}_{\mathbf{u}}(t)+\mathbf{H}_{\mathbf{g}}{ }^{\mathrm{T}} \mathbf{g}+\mathbf{H}_{\mathbf{J}_{1,2}}{ }^{\mathrm{T}} \mathbf{w}_{1,2}$ 
In this equation (1), $\mathbf{q}$ is the $R \times 1$ column matrix (vector) of the modal coordinates ( $R$ is the number of modes), $\boldsymbol{\Lambda}$ is the diagonal matrix of the squares of the eigenfrequencies $\omega_{1}, \ldots, \omega_{R}$. As shown in Figure 1, the HP rotor is supported by two unsupported SFD bearings and their forces are contained in $\mathbf{f}_{\mathbf{v}_{n}}\left(\mathbf{v}_{n}, \dot{\mathbf{v}}_{n}, t\right)$, which are non-linear functions. The inputs are the instantaneous displacements and velocities of the journal centres (marked ' $J_{1}$ or 2 ' in Figure 1 ) relative to the centres of their housings (marked ' $\mathrm{B}_{1 \text { or } 2}$ ' in Figure 1). The vector $\mathbf{v}_{n}$ contains the instantaneous Cartesian $(x, y)$ displacements of $\mathrm{J}_{n}$ relative to $\mathrm{B}_{n}$, and can be expressed as:

$\mathbf{v}_{n}=\left[\begin{array}{l}x_{\mathrm{rel}, n} \\ y_{\mathrm{rel}, n}\end{array}\right]=\mathbf{H}_{\mathbf{v}_{n}} \mathbf{q}$

where, the term $\mathbf{H}_{\mathbf{v}_{n}}$ is a $2 n \times R$ matrix, the columns of which are:

$\boldsymbol{\Psi}_{\mathbf{v}_{n}}^{(r)}=\boldsymbol{\Psi}_{\mathbf{J}_{n}}^{(r)}-\boldsymbol{\Psi}_{\mathbf{B}_{n}}^{(r)}, \quad r=1 \ldots R$

where, the subscript $n$ refers to each SFD bearing case, with $n=1$ and $n=2$ denoting the unsupported SFD bearing (lefthand side) and the SFD bearing (right-hand side), respectively. Let $\mathbf{v}_{n}$ then be the vector of the instantaneous Cartesian $(x, y)$ displacements of the SFD journal relative to $\mathrm{J}_{n}$ relative to $\mathrm{B}_{n}$. The vectors $\boldsymbol{\Psi}_{\mathbf{J}_{n}}^{(r)}, \boldsymbol{\Psi}_{\mathbf{B}_{n}}^{(r)}$ are the mass-normalized eigenvectors defining the $x, y$ displacements of the squeeze-film at each terminal $\mathrm{J}_{n}$ and $\mathrm{B}_{n}$, respectively, in mode no. $r(r=1 \ldots R)$. Since both SFD bearing cases are unsupported, the eccentric mean position is completely determined by the equilibrium between the equivalent rotor weight at each $\mathrm{J}_{n}$ and the mean SFD forces. Therefore, for this case the effect of the static loading on each journal $\mathrm{J}_{n}$ in $x, y$ directions is considered in the $4 \times 1$ vector $\mathbf{w}_{1,2}$, which is multiplied by the transpose of matrix $\mathbf{H}_{\mathbf{J}_{1,2}}{ }^{\mathrm{T}}$, whose columns are the eigenvectors $\boldsymbol{\Psi}_{\mathbf{I}_{1,2}}^{(r)}$.

The unbalance forces are contained in vector $\mathbf{f}_{\mathbf{u}}$, expressed as follows:

$\mathbf{f}_{\mathbf{u}}=\left[\begin{array}{lllllll}U_{x_{1}} & U_{y_{1}} & U_{x_{2}} & U_{y_{2}} & \cdots & U_{x_{n_{\text {unb }}}} & U_{y_{n_{\text {unb }}}}\end{array}\right]^{\mathrm{T}}$

where, $U_{x_{k}}, U_{y_{k}} k=1,2,3, \ldots, n_{\text {unb }}$ are the unbalance forces presumed to be located at disk positions $k=1,2,3, \ldots, n_{\mathrm{unb}}$ :

$U_{x_{k}}=U_{k} \Omega^{2} \sin \left(\Omega t+\gamma+\phi_{k}\right)$

$U_{y_{k}}=-U_{k} \Omega^{2} \cos \left(\Omega t+\gamma+\phi_{k}\right)$

where, $U_{k}$ denotes the unbalance (in $\mathrm{Kg} \mathrm{m}$ ) positioned at disk no. $k$, and its angular location $\phi_{k}$ from a marker on the rotor, it is displaced by an angle $\Omega t$ from the phase probe (stationary), that is at an angle $\gamma$ from the downward vertical as shown in Figure 3(a)). In Figure 3(b) is showed the unbalance distribution over the HP rotor at ten unbalance positions matching with the disks of the system, including coupling disks (Figure 1).

(a)

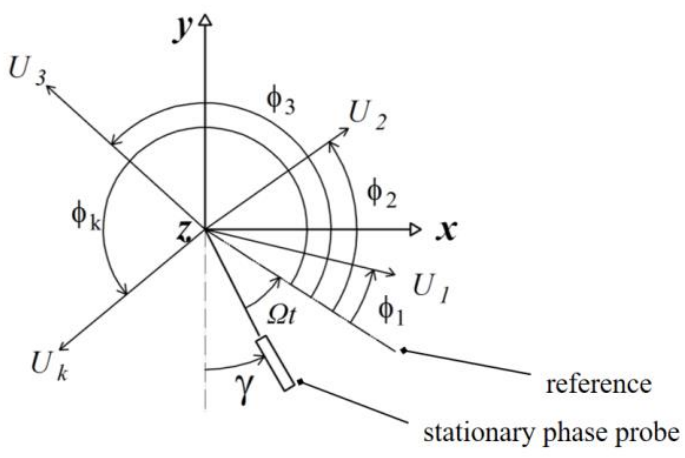

(b)

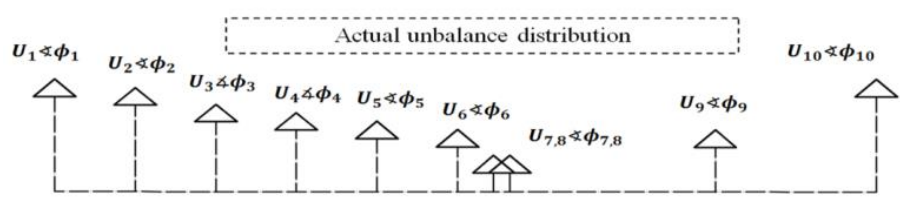

Figure 3. The circular rotation of the unbalance forces: (a) angular allocation of unbalance with the stationary phase probe and the reference phasor; (b) ten locations of unbalance distribution [18]

As in previous studies $[18,21,23,24]$ gyroscopic effects are discretised at a number position $(G)$ on the rotor. So, the gyroscopic effects are contained in vector $\mathbf{g}$ and considered like the forces $\mathbf{f}_{\mathbf{v}_{n}}$ and $\mathbf{f}_{\mathbf{u}}$, as external to the non-linear rotating part.

$\mathbf{g}=\mathbf{P} \dot{\boldsymbol{\theta}}$

where $\mathbf{g}=\left[\begin{array}{lllll}M_{1} & N_{1} & \ldots & M_{G} & N_{G}\end{array}\right]^{\mathrm{T}}, \boldsymbol{\theta}=\left[\begin{array}{llllll}\beta_{1} & \alpha_{1} & \ldots & \beta_{G} & \alpha_{G}\end{array}\right]^{\mathrm{T}} \quad$ (as the system coordinate shown in Figure 1). $\mathbf{P}=\Omega \operatorname{diag}\left\{-I_{1}, I_{1}, \ldots \ldots,-I_{G}, I_{G}\right\}$, where $I_{p}$ refers to the polar position $p$ of the moment of inertia $(p=1, \ldots, G)$. The modal matrices $\mathbf{H}_{\mathbf{u}}$ include columns $\left(2 n_{\text {unb }} \times R\right)$ refer to by $\boldsymbol{\Psi}_{\mathbf{u}}^{(r)}$ and the $\mathbf{H}_{\mathbf{g}}$ comprise $\boldsymbol{\Psi}_{\mathbf{g}}^{(r)}$ as $2 G \times R$ columns, while the eigenvectors are considered at the positions and directions of the notations in $\mathbf{f}_{\mathbf{u}}, \mathbf{g}$.

It is noted that the basic approach used in eq. (1) i.e. modal equations of the linear part acted on by "external" (i.e. right hand side) excitations (including the nonlinear bearing forces and gyroscopic moments), was first introduced by Adams [26] and has been successfully used in experimentally validated studies of flexible rotors with highly nonlinear unsupported SFDs e.g. [27].

\subsection{GENERATION OF SFD FORCES FROM CASING VIBRATION RESPONSE}

As already undertaken in [5,18], the "implicit" inverse operator is constructed using the Receptance Harmonic Balance Method (RHBM). This enables transposing eq. (1) in the frequency domain and also, by using it any selected physical degrees of freedom can be condensed from modal 
coordinates [21]. The RHBM equations are inverted in the present study to generate the SFD forces from external casing vibration data, which will then be employed as network inputs. The inversion process assumes periodic vibration, where the fundamental frequency is equal to the excitation-rotational speed $\Omega$. Hence, in the case of the vibration readings of velocity at each casing location $P_{i}, i=1 \ldots n_{\mathrm{s}}$ in the $x, y$ directions, this is defined as an $2 \times 1$ vector [5]:

$\mathbf{z}_{\mathbf{p}_{i}}=\sum_{s=1}^{m} \mathbf{z}_{\mathbf{p}_{i_{\mathrm{c}}}}^{(s)} \cos (s \Omega t)+\mathbf{z}_{\mathbf{p}_{i_{\mathrm{s}}}}^{(s)} \sin (s \Omega t)$

where

$\mathbf{z}_{\mathbf{p}_{i}}=\dot{\mathbf{x}}_{\mathbf{p}_{i}}, \quad \mathbf{x}_{\mathbf{p}_{i}}=\left[\begin{array}{l}x_{\mathbf{p}_{i}}(t) \\ y_{\mathbf{p}_{i}}(t)\end{array}\right]$

and

$\mathbf{x}_{\mathbf{p}_{i}}(t)=\overline{\mathbf{x}}_{\mathbf{p}_{i}}+\sum_{s=1}^{m} \mathbf{x}_{\mathbf{p}_{i_{\mathrm{c}}}}^{(s)} \cos (s \Omega t)+\mathbf{x}_{\mathbf{p}_{i_{\mathrm{S}}}}^{(s)} \sin (s \Omega t)$

With reference to Equation (1), defining

$\mathbf{f}_{\mathbf{v}}=\left[\begin{array}{l}\mathbf{f}_{\mathbf{v}_{1}}\left(\mathbf{v}_{1}, \dot{\mathbf{v}}_{1}, t\right) \\ \mathbf{f}_{\mathbf{v}_{2}}\left(\mathbf{v}_{2}, \dot{\mathbf{v}}_{2}, t\right)\end{array}\right]$

the $4 \times 1$ vector $\mathbf{f}_{\mathbf{v}}$ can therefore also be expressed as:

$\mathbf{f}_{\mathbf{v}}(t)=\overline{\mathbf{f}}_{\mathbf{v}}+\sum_{s=1}^{m} \mathbf{f}_{\mathbf{v}_{\mathbf{c}}}^{(s)} \cos (s \Omega t)+\mathbf{f}_{\mathbf{v}_{\mathrm{s}}}^{(s)} \sin (s \Omega t)$

The casing vibration readings $\mathbf{z}_{\mathbf{p}_{i}}$ are the result of the actual unbalance distribution shown in Figure 3, which is described by the actual excitation vector $\mathbf{f}_{\mathbf{u}}$ (eq. (4)). As previously reported in [7], in the case where there is no linear connection between the rotor and the casing, the excitation from the unbalance effect only appears implicitly in the RHBM equation at the casing through the motion-dependent SFD forces. In the current study case, $\mathrm{P}_{1}$ and $\mathrm{P}_{2}$ were chosen as the reference locations, since these conveniently coincide with the axial locations of the SFDs.

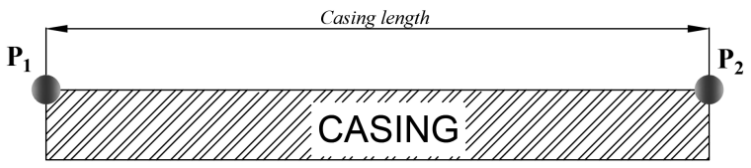

Figure 4. The locations of the sensor over the casing.

The approach used here the same as that of [5], whereby RHBM is used to express each harmonic of the vibration response readings measured at two locations $P_{1}$ and $P_{2}$ (see Figure 4) in terms of the corresponding harmonic of the bearing (SFD) forces $-\mathbf{f}_{\mathbf{v}}$ acting on the casing-bearing housings, as follows:

$$
\left[\begin{array}{c}
\mathbf{z}_{\mathbf{p}_{\mathbf{1}_{\mathrm{S}}}}^{(s)} \\
\mathbf{z}_{\mathbf{p}_{\mathbf{1}_{\mathrm{C}}}^{(s)}}^{(s)} \\
\mathbf{z}_{\mathbf{p}_{\mathbf{2}_{\mathrm{S}}}}^{(s)} \\
\mathbf{z}_{\mathbf{p}_{\mathbf{3}_{\mathrm{C}}}}^{(s)}
\end{array}\right]=\left[\begin{array}{cc}
-\mathbf{M}_{\mathbf{p}_{\mathbf{1}} \mathbf{B}}(s \Omega) & \mathbf{0} \\
\mathbf{0} & \mathbf{M}_{\mathbf{p}_{\mathbf{1}} \mathbf{B}}(s \Omega) \\
-\mathbf{M}_{\mathbf{p}_{\mathbf{B}} \mathbf{B}}(s \Omega) & \mathbf{0} \\
\mathbf{0} & \mathbf{M}_{\mathbf{p}_{2} \mathbf{B}}(s \Omega)
\end{array}\right]\left[\begin{array}{c}
-\mathbf{f}_{\mathbf{v}_{\mathbf{c}}}^{(s)} \\
-\mathbf{f}_{\mathbf{v}_{\mathbf{s}}}^{(s)}
\end{array}\right], s=1 \ldots m .
$$

where

$$
\mathbf{M}_{\mathbf{p}_{i} \mathbf{B}}=s \Omega \mathbf{C}_{\mathbf{p}_{i} \mathbf{B}}(s \Omega), \quad \mathbf{C}_{\mathbf{p}_{i} \mathbf{B}}(s \Omega)=\sum_{r=1}^{R} \frac{\left.\boldsymbol{\Psi}_{\mathbf{P}_{i}}^{(r)} \boldsymbol{\Psi}_{\mathbf{B}}^{(r)}\right)^{\mathrm{T}}}{\omega_{r}^{2}-(s \Omega)^{2}}
$$

and, with reference to eq. (15) and Figure 1:

$\boldsymbol{\Psi}_{\mathbf{B}}^{(r)}=\left[\begin{array}{l}\boldsymbol{\Psi}_{\mathbf{B}_{1}}^{(r)} \\ \boldsymbol{\Psi}_{\mathbf{B}_{2}}^{(r)}\end{array}\right]$

By inverting eq. (13) at each harmonic, the SFD forces can be obtained from external casing vibration response. These SFD forces are denoted as $\breve{\mathbf{f}}_{\mathbf{v}_{\mathbf{c}}}^{(s)}, \breve{\mathbf{f}}_{\mathbf{v}_{\mathrm{S}}}^{(s)}$ and obtained as follows:

$$
\left[\begin{array}{c}
\breve{\mathbf{f}}_{\mathbf{v}_{\mathbf{c}}}^{(s)} \\
\breve{\mathbf{f}}_{\mathbf{v}_{\mathrm{S}}}^{(s)}
\end{array}\right]=-\left[\begin{array}{cc}
-\mathbf{M}_{\mathbf{p}_{\mathbf{1}} \mathbf{B}}(s \Omega) & \mathbf{0} \\
\mathbf{0} & \mathbf{M}_{\mathbf{p}_{\mathbf{1}} \mathbf{B}}(s \Omega) \\
-\mathbf{M}_{\mathbf{p}_{2} \mathbf{B}}(s \Omega) & \mathbf{0} \\
\mathbf{0} & \mathbf{M}_{\mathbf{p}_{2} \mathbf{B}}(s \Omega)
\end{array}\right]^{-\mathbf{1}}\left[\begin{array}{c}
\mathbf{z}_{\mathbf{p}_{1}}^{(s)} \\
\mathbf{z}_{\mathbf{p}_{\mathbf{1}}}^{(s)} \\
\mathbf{z}_{\mathbf{p}_{\mathbf{2}_{\mathrm{S}}}}^{(s)} \\
\mathbf{z}_{\mathbf{p}_{\mathbf{2}_{\mathrm{C}}}}^{(s)}
\end{array}\right], s=1 \ldots \ldots m
$$

These are then used to obtain ${ }^{d} \breve{f}_{\mathbf{v}}(t)$ of the periodic time history of ${ }^{\mathrm{d}} \mathbf{f}_{\mathbf{v}}(t)$, which denotes the dynamic part of $\mathbf{f}_{\mathbf{v}}(t)$ i.e. excluding the mean term in eq. (12):

$\mathrm{d}_{\mathbf{f}_{\mathbf{v}}}(t)=\sum_{s=1}^{m} \breve{\mathbf{f}}_{\mathbf{v}_{\mathbf{c}}}^{(s)} \cos (s \Omega t)+\breve{\mathbf{f}}_{\mathbf{v}_{\mathbf{s}}}^{(s)} \sin (s \Omega t)$

$\mathrm{d}_{\mathbf{f}_{\mathbf{v}}}(t)$ contains the dynamic parts of the time histories of the $x, y$ forces at the two SFDs:

$\mathrm{d}_{\mathrm{f}_{\mathbf{v}}}(t)=\left[\begin{array}{l}{ }^{\mathrm{d}} \breve{\mathbf{f}}_{\mathbf{v}_{\mathbf{1}}}(t) \\ { }_{\mathrm{d}} \breve{\mathbf{f}}_{\mathbf{v}_{\mathbf{2}}}(t)\end{array}\right]$

As in [5], $\mathrm{d}(\mathrm{)}$ refers to the exclusion of the mean component from the quantity ( ), as in eq. (18), whereas ( $)$ denotes the quantity ( ) based on the inversion of Equation (17). As described in the following section, for each SFD no. $n$, a trained topology (network) can be utilised to obtain a prediction $\breve{\mathbf{v}}_{n}(t)$ of the $x, y$ journal displacements relative to housing, corresponding to a given input forces ${ }^{d_{\mathbf{f}_{v}}}(t)$. The process is shown in Figure 5.

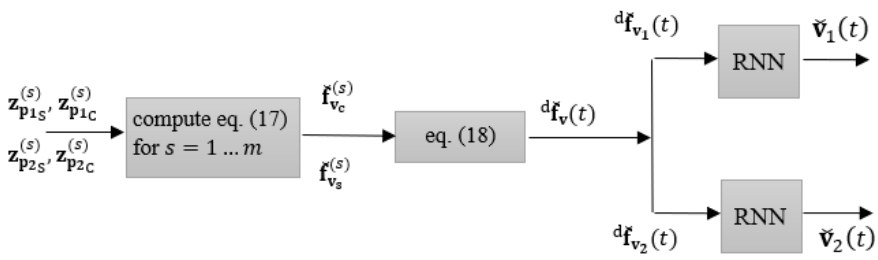

Figure 5. The Fourier coefficients of vibration of the journal estimated at the two SFDs using casing vibration readings at two locations $\left(\mathrm{P}_{1}\right.$ and $\left.\mathrm{P}_{2}\right)$

\section{FULLY IMPLICIT RNN SFD INVERSE MODEL}

As reported in $[5,7,19]$, the instantaneous SFD bearing is a function of the instantaneous displacements/velocities, the forward model does not have memory and "memoryless" identification methods like Chebyshev [10] or static neural 
networks [12]. In the case of the inverse SFD model, consideration of the Reynolds Equation shows that it is not possible to determine the SFD force simply from the instantaneous values of the journal displacement and velocity. However, it should be possible to determine a time history of $\mathbf{v}_{n}\left(t_{k}\right)$ that corresponds to a given input time history of ${ }^{d} \mathbf{f}_{\mathbf{v}_{n}}(t)$ by considering that the current value of the output $\mathbf{v}_{n}\left(t_{k}\right)$ as a function of the past values of both the input ${ }^{\mathrm{d}} \mathbf{f}_{\mathbf{v}_{n}}\left(t_{k}\right)$ and the output $\mathbf{v}_{n}\left(t_{k}\right)$ itself. Therefore, a time series modelling scheme based on the Nonlinear Autoregressive Exogenous (NARX) scheme was employed due to its system memory.

With reference to Figure 6, the RNN's external input is the SFD force signal ${ }^{d} \mathbf{f}_{\mathbf{v}_{n}}\left(t_{k}\right)$, where $t_{k}=(k-1) \Delta, k=1,2, \ldots$, denotes discrete times, $\Delta$ being the sampling time resolution. It should be noted that, as in previous work [5], the mean is again excluded since the SFD force inputs were derived from the casing vibration readings, as per Figure 4. Also, the subscript "a" is dropped from the following notation for the avoidance of doubt. The network's output estimate for $\mathbf{v}_{n}\left(t_{k}\right)$ is designated by $\check{\mathbf{v}}_{n}\left(t_{k}\right)$. The inputs and outputs are normalised for network use to a range of [-1,1] [28], where

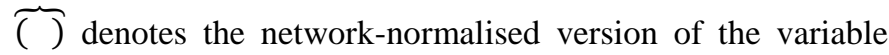
( ). System memory is therefore considered through the tapped delay lines (TDLs), which are channels containing past values of the input and output (feedback) signals.

With reference to Figure 6 and [7], let $S^{(j)}$ be the number of neurons in the $j^{\text {th }}$ layer, $J$ be the total number of layers, whilst $M_{1}$ and $M_{2}$ are the number of delays in TDL no.1 and TDL no.2, respectively. If $\mathbf{a}_{k}^{(j)}$ is the $S^{(j)} \times 1$ vector comprising the signal outputs of the $j^{\text {th }}$ layer, then:

$\mathbf{a}_{k}^{(J)}=\widetilde{\mathbf{v}}_{n}\left(t_{k}\right)$

$\mathbf{a}_{k}^{(1)}=\mathbf{g}^{(1)}\left(\mathbf{W}^{(1)} \mathbf{p}_{k}+\mathbf{b}^{(1)}\right)$

$\mathbf{a}_{k}^{(j)}=\mathbf{g}^{(j)}\left(\mathbf{W}^{(j)} \mathbf{a}_{k}^{(j-1)}+\mathbf{b}^{(j)}\right), j=2, \ldots . J$

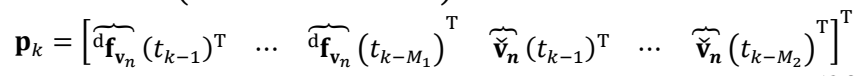

$\overbrace{\mathbf{d}_{\mathbf{v}_{n}}}\left(t_{k-m}\right), \widetilde{\mathbf{v}}_{\boldsymbol{n}}\left(t_{k-m}\right)=\mathbf{0}, \quad t_{k-m}<t_{1}, m=1, \ldots . \max \left(M_{1}, M_{2}\right)$

where, $\mathbf{W}^{(j)}$ and $\mathbf{b}^{(j)}$ are respectively the matrix of weights and vector of biases of the $j^{\text {th }}$ layer, whilst $\mathbf{g}^{(j)}()$ is a vector operator comprising the transfer functions of the neurons of the $j^{\text {th }}$ layer.

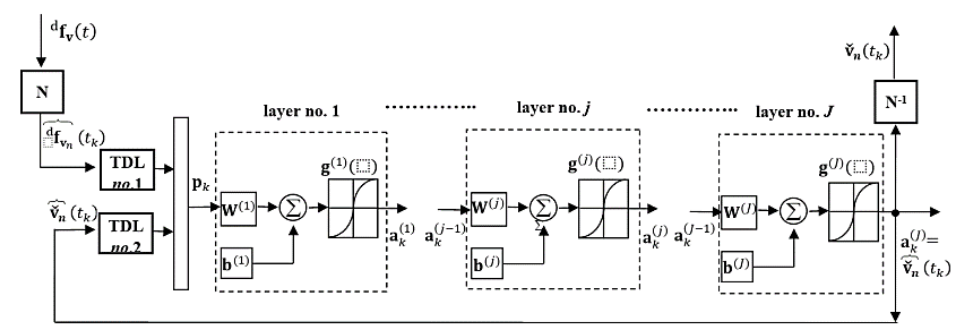

Figure 6. RNN architecture for inverse SFD model [5].
As previously performed in [5], the RNN for the inverse model of each of SFD in Figure 2 was implemented in general-purpose technical computing software that has a neural network facility [29]. For a given user-prescribed architecture, the RNN was subjected to input/output training data. The training procedure used was that of the LevenbergMarquardt optimization method [29] to determine the optimal weights and biases that minimize the mean square value of the error (difference) $\delta$ between the output of the network $\check{\mathbf{v}}_{n}\left(t_{k}\right)$ and the actual data (i.e. target) output $\mathbf{v}_{n}\left(t_{k}\right)$ for a given input $\mathrm{d}_{\mathbf{f}_{\mathbf{v}_{n}}}\left(t_{k}\right)$.

\subsection{GENERATION OF TRAINING DATA AND RNN DETAILS}

For the present study, a procedure reported in work by [5] was employed, where the training data are generated by replacing the unbalance excitation in Figure 2 with "circular chirp excitation" applied to the rotor at two locations, as shown in Figure 7. This excitation at each location, $i=1,2$, consists of a pair of forces $P_{x_{i}}(t)$ and $P_{y_{i}}(t)$ in the $x$ and $y$ directions, with time histories in the form of orthogonallyphased harmonic functions of equal and constant amplitude as well as steadily-increasing frequency (chirp signals) [5]:

$$
\begin{aligned}
& P_{x_{i}}(t)=A \sin \left[2 \pi\left\{f_{\text {init }} t+\frac{\left(f_{\text {end }}-f_{\text {init }}\right)}{\left(t_{\text {end }}-t_{\text {init }}\right)}\left(\frac{t^{2}}{2}\right)\right\}\right] \\
& P_{y_{i}}(t)=-A \cos \left[2 \pi\left\{f_{\text {init }} t+\frac{\left(f_{\text {end }}-f_{\text {init }}\right)}{\left(t_{\text {end }}-t_{\text {init }}\right)}\left(\frac{t^{2}}{2}\right)\right\}\right]
\end{aligned}
$$

where, $f_{\text {init }}$ and $f_{\text {end }}$ are the excitation frequencies of the frequency sweep at the beginning $\left(t=t_{\text {init }}\right)$ and end $(t=$ $\left.t_{\text {end }}\right)$, respectively. Accordingly, the forward problem described in Section 3.1 was solved with the unbalance excitation term $\mathbf{H}_{\mathbf{u}}{ }^{\mathrm{T}} \mathbf{f}_{\mathbf{u}}$ in eq. (1) being replaced by $\mathbf{H}_{\text {exc }}{ }^{\mathrm{T}} \mathbf{f}_{\text {exc }}$, where:

$$
\mathbf{f}_{\mathbf{e x c}}=\left[\begin{array}{llll}
P_{x_{1}}(t) & P_{y_{1}}(t) & P_{x_{2}}(t) & P_{y_{2}}(t)
\end{array}\right]^{\mathrm{T}}
$$

The modal matrix $\mathbf{H}_{\text {exc }}(4 \times R)$ has columns $\boldsymbol{\Psi}_{\text {ext }}^{(r)}, r=$ $1 \ldots R$ that are the eigenvectors evaluated at degrees of freedom corresponding to the locations and directions of the entries in $\mathbf{f}_{\text {exc }}$. The excitation amplitude $A$ (Equations (21a, b)) was set at $4000 \mathrm{~N}$ at both excitation locations over the rotor. The frequency sweep was performed over an interval of 0 to $30 \mathrm{~s}$ and covered frequencies in the range $133.33 \mathrm{~Hz}$ $(8000 \mathrm{rpm})$ to $266.66 \mathrm{~Hz}(16000 \mathrm{rpm})$. The time histories of $\mathbf{v}_{n}(t)$ and $\mathbf{f}_{\mathbf{v}_{n}}(t)$ were computed at each time step, as described in Section 3.1. For the purpose of creating the training data sets, the variable-step integration data were interpolated at equal time intervals $t=t_{k}=(k-1) \Delta$, where $k=1,2, \ldots$ The data for $\mathbf{f}_{\mathbf{v}_{n}}\left(t_{k}\right)$ over $0 \leq t_{k} \leq 30$ were then divided into blocks of 100 sampling points each and the mean of the block was removed, thus creating the required input training data ${ }^{\mathrm{d}} \mathbf{f}_{\mathbf{v}_{n}}\left(t_{k}\right)$. It should be noted that the mean of the 
target network output $\mathbf{v}_{n}\left(t_{k}\right)$ is retained. The resulting training data can be expressed as follows,

$$
\begin{aligned}
& \mathbf{v}_{n}\left(t_{k}\right)=\left[x_{\operatorname{rel}_{n}}\left(t_{k}\right) \quad y_{\mathrm{rel}_{n}}\left(t_{k}\right)\right]^{\mathrm{T}} \\
& { }^{\mathrm{d}} \mathbf{f}_{\mathbf{v}_{n}}\left(t_{k}\right)=\left[\begin{array}{ll}
{ }^{\mathrm{d}} Q_{x_{n}}\left(t_{k}\right) & { }^{\mathrm{d}} Q_{y_{n}}\left(t_{k}\right)
\end{array}\right]^{\mathrm{T}}
\end{aligned}
$$

and shown in Figure 8 for the left-hand side SFD $(n=1)$ and Figure 9 for the right-hand side SFD $(n=2)$. With regard to the force and displacement variation in Figures 8 and 9, it is noted that an unsupported SFD relies on vibration of the journal relative to the housing to generate lift to overcome the static load (rotor weight). As the frequency increases, the vibration amplitude decreases and the mean position in the $y$ direction correspondingly decreases. The low amplitude vibration in this condition can still generate a significant dynamic force due to the squeezing of the oil in the restricted space at the bottom of the annular clearance (note that the graphs in Figures 8 and 9 show the dynamic part of the SFD forces).

As already stated, two RNNs were trained. The first one was related with the left hand-side (LH) SFD network, trained using the data in Figure 8, had three layers, with 4, 15, 2 neurons, respectively and $M_{1}=2$ delays for the input and $M_{2}=2$ for the feedback. The second one was related to the right hand-side (RH) SFD network, trained using data in Figure 9, had also three layers with 3,6, 2 neurons, respectively and $M_{1}=2$ delays for the input and $M_{2}=2$ for the feedback. In both cases, tan-sigmoid transfer functions [17, 28] were used for the neurons, except for those in the final (output) layer, where linear transfer functions were deployed.

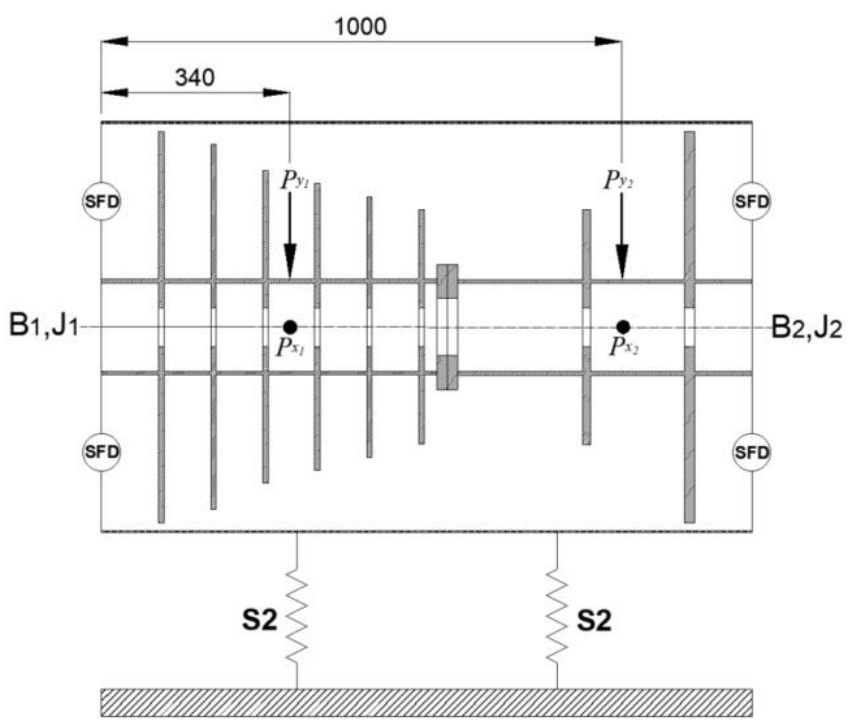

Figure 7. Chirp type excitation at two locations over the HP rotor (dimensions in $\mathrm{mm}$ ).
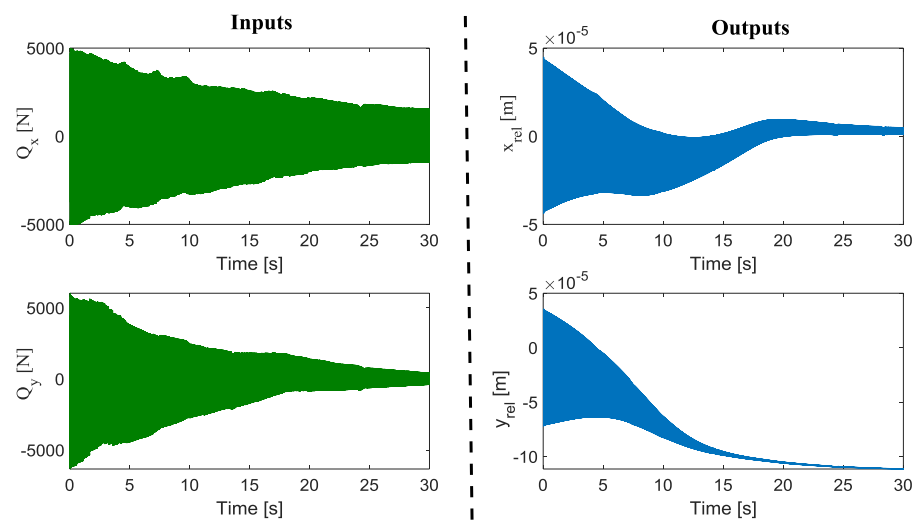

Figure 8. Input/Output data set used to train the left-hand side SFD $(n=1)$ network.
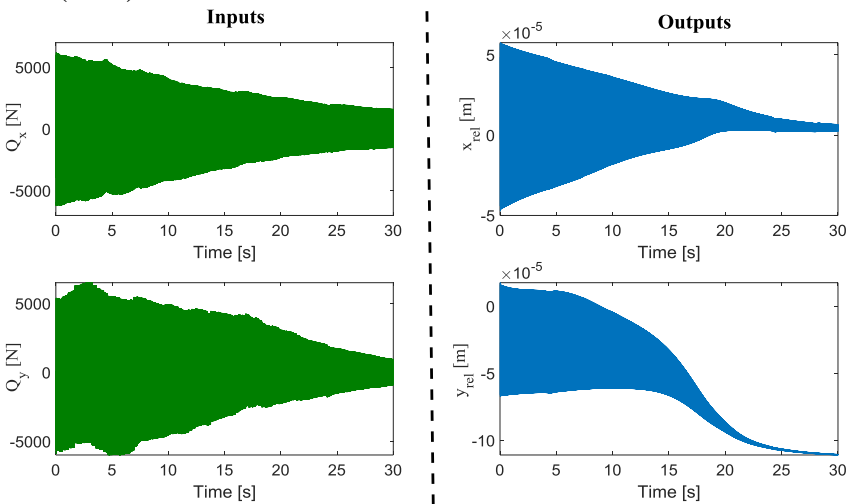

Figure 9. Input/Output data set used to train the right-hand side SFD $(n=2)$ network.

\section{STUDY CASES AND DISCUSIONS}

\subsection{GENERATION OF SIMULATED VIBRATION DATA}

Following previous works $[5,18]$, the current case study is also based on simulated casing vibration data. The simulated data were then computed by solving equation (1) in the time domain for the SFD system described in Figure 1. The standard solution approach is to express eq. (1) into $2 R$ first order differential equation and apply robust implicit step-bystep integration to calculate $\mathbf{q}_{k}$ and $\dot{\mathbf{q}}_{k}$ for known $\mathbf{q}_{k-1}, \dot{\mathbf{q}}_{k-1}$, and a known excitation vector $\mathbf{f}_{\mathbf{u}}$, where $\mathbf{q}_{k}=\mathbf{q}\left(t_{k}\right), \dot{\mathbf{q}}_{k}=$ $\dot{\mathbf{q}}\left(t_{k}\right)$, and $t_{k}=t_{k-1}+\Delta t_{k}$. As done in $[5,18]$, it was used a conventional implicit integration algorithms ode23s@ (The modified Rosenbrock algorithm ) available in Matlab@ [30]. The response time histories at the physical degrees of freedom were then computed from $\mathbf{q}_{k}$ using the appropriate modal transformation matrices $[5,18]$ e.g. as done in eq. (2).

The SFD forces were obtained by using the more realistic approach as in $[5,18,24]$. Therefore, the SFDs considered for this study were single-land and end-fed with oil of viscosity 0.0049 Nsm-2 at a pressure of 3 bar (gauge), as in $[5,17,18,21,31]$. The bearing diameter and radial clearance were $220 \mathrm{~mm}, 0.12 \mathrm{~mm}$ respectively, and the land length was $26 \mathrm{~mm}$. Through the integration process, the instantaneous pressure distribution across each oil film were approximated by a combination of the short and long bearing expressions through an "end leakage" factor of 0.1 , which quantifies the 
degree of sealing at outlet of the SFDs [22]. This distribution was truncated below a cavitation pressure of absolute zero ($101.325 \mathrm{kPa}$ ) and numerically integrated across the oil film to generate SFD forces.

The testing cases involves three distinct unbalance distributions which are presented in Table 1. The seeded unbalance planes (see Figure 3) are placed at each disk shown in Figure 1. For the present study, the reference phasor probe position $\gamma$ (Figure $3(\mathrm{a})$ ) is taken to be $0^{\circ}$.

\begin{tabular}{|c|c|c|c|c|c|c|c|c|c|c|}
\hline$k$ & 1 & 2 & 3 & 4 & 5 & 6 & 7 & 8 & 9 & 10 \\
\hline \multicolumn{10}{|c|}{ Case 1 } \\
\hline$U_{k}$ & 0.1 & 0.5 & 0.1 & 1.0 & 0.5 & 1.0 & 0.1 & 1.5 & 0.3 & 1.5 \\
\hline$\phi_{k}$ & 0 & 110 & 305 & 85 & 195 & 145 & 75 & 10 & 215 & 330 \\
\hline \multicolumn{10}{|c|}{ Case 2 } \\
\hline$U_{k}$ & 1.2 & 0.3 & 1.1 & 1.0 & 0.9 & 1.5 & 0.6 & 1.5 & 0.8 & 1.5 \\
\hline$\phi_{k}$ & 30 & 140 & 205 & 305 & 15 & 170 & 90 & 10 & 200 & 350 \\
\hline \multicolumn{10}{|c|}{ Case 3 } \\
\hline$U_{k}$ & 1.1 & 1.5 & 2.9 & 1.5 & 1.7 & 1.5 & 2.6 & 1.5 & 1.8 & 1.9 \\
\hline$\phi_{k}$ & 15 & 160 & 15 & 110 & 75 & 265 & 350 & 220 & 95 & 190 \\
\hline
\end{tabular}

Table 1. Cases of seeded unbalance distributions

The forward calculation considered $R=14$ modes (i.e. 7 modes in each of the horizontal and vertical planes). Both SFD bearings are unsupported, therefore, the eccentric mean position of the journal is determined by the equilibrium between the equivalent rotor weight at each $\mathrm{J}_{n}$ and the mean SFD forces.

As earlier described, $\mathrm{P}_{1}$ and $\mathrm{P}_{2}$ are chosen as the reference locations since these conveniently coincide with the centres of the SFD housings (marked ' $\mathrm{B}_{1}$ or 2 ' in Figure 1). Therefore, the steady-state part of the time histories of $\mathbf{q}$ and $\dot{\mathbf{q}}$ were then used to calculate the simulated velocity sensor readings at $\mathrm{B}_{1 \text { or } 2}$ as follows:

$\dot{\mathbf{x}}_{\mathbf{B}_{n}}=\mathbf{H}_{\mathbf{B}_{n}} \dot{\mathbf{q}}=\dot{\mathbf{x}}_{\mathbf{P}}=\mathbf{H}_{\mathbf{P}} \dot{\mathbf{q}}$

where $\dot{\mathbf{x}}_{\mathbf{B}_{n}}$ is the $2 n \times 1$ vector of velocity responses, alternately in the $x, y$ directions at $n_{\mathrm{s}}$ locations along the casing (an $x$ vibration sensor and a $y$ vibration housing at each SFD location as illustrated in Figure 4):

$\dot{\mathbf{x}}_{\mathbf{B}_{n}}=\left[\begin{array}{l}\dot{\mathbf{x}}_{\mathbf{B}_{1}} \\ \dot{\mathbf{x}}_{\mathbf{B}_{2}}\end{array}\right]=\dot{\mathbf{x}}_{\mathbf{P}}=\left[\begin{array}{l}\dot{\mathbf{x}}_{\mathbf{P}_{1}} \\ \dot{\mathbf{x}}_{\mathbf{p}_{2}}\end{array}\right]$

$\mathbf{H}_{\mathbf{B}_{n}}$ is the $2 n \times R$ matrix whose $R$ columns are the eigenvectors evaluated at the sensor locations $\mathbf{B}_{n}$, alternately in the $x, y$ directions.

$\mathbf{H}_{\mathbf{B}_{n}}=\left[\begin{array}{ccc}\boldsymbol{\Psi}_{\mathbf{B}_{1}}^{(1)} & \cdots & \boldsymbol{\Psi}_{\mathbf{B}_{1}}^{(R)} \\ \boldsymbol{\Psi}_{\mathbf{B}_{2}}^{(1)} & \cdots & \boldsymbol{\Psi}_{\mathbf{B}_{2}}^{(R)}\end{array}\right]=\mathbf{H}_{\mathbf{P}}=\left[\begin{array}{ccc}\boldsymbol{\Psi}_{\mathbf{P}_{1}}^{(1)} & \ldots & \boldsymbol{\Psi}_{\mathbf{P}_{1}}^{(R)} \\ \boldsymbol{\Psi}_{\mathbf{P}_{2}}^{(1)} & \cdots & \boldsymbol{\Psi}_{\mathbf{P}_{2}}^{(R)}\end{array}\right]$
In contrast to previous work [5], this study considered three different unbalance distributions. Therefore, the aim of this work is to determine the degree of accuracy for estimating the relative displacements at the SFD journals from the external vibration readings based on RNN modelling. The following sections then show two types of studies, the first one deals with the performance of each inverse SFD models at one velocity speed. The second will show performance over 27 operational speeds.

\subsection{STUDY CASE 1: RNN-PERFORMANCE AT ONE OPERATIONAL SPEED}

The present study case was designed to compare the signals $\mathrm{d}_{\mathbf{f}_{\mathbf{v}_{n}}}(t), \breve{\mathbf{v}}_{n}(t)$ with the "true" signals ${ }^{\mathrm{d}} \mathbf{f}_{\mathbf{v}_{n}}(t), \mathbf{v}_{n}(t)$ for each SFD no. $n=1,2$, respectively. The true ones were obtained from the steady-stated forward solution as described in section 3.1 .

Figures 10(a-c) refer to the Left-Hand (LH ) side SFD bearing, as illustrated in Figure 1, and thus compare the signals ${ }^{\mathrm{d}} \breve{\mathbf{f}}_{\mathbf{v}_{1}}(t), \breve{\mathbf{v}}_{1}(t)$ with the "true" signals ${ }^{\mathrm{d}} \mathbf{f}_{\mathbf{v}_{1}}(t), \mathbf{v}_{1}(t)$ respectively. These results are obtained at one operational speed which is 11250 RPM. Unlike previous work [5], the current study involves three different unbalance conditions shown in Table 1. Hence, these unbalance conditions are very different from those used in training the RNNs (section 4.1). The most significant difference is that the training data were obtained under no rotation conditions and the validation data were obtained under rotating conditions. Following the procedure described in Figure 5, the first column of each of the Figures 10(a-c) show a comparison of the SFD forces within ${ }^{d} \breve{f}_{\mathbf{v}_{1}}(t)$ (solid lines) with the true ones within ${ }^{d} \mathbf{f}_{\mathbf{v}_{1}}(t)$ (dotted lines) ( $x, y$ forces shown in upper, lower rows respectively). It is seen that the calculation of eq. (18) is truly closed to the true signal.

Turning now to the second column of each of the Figures $10(\mathrm{a}-\mathrm{c})$, where is compared the $x, y$ relative displacements within $\breve{\mathbf{v}}_{1}(t)$ which is the RNN output for the input ${ }^{d} \breve{\mathbf{f}}_{\mathbf{v}_{1}}(t)$, with the true relative displacement signals within $\mathbf{v}_{1}(t)$ (dotted lines) ( $x, y$ displacement time histories shown in upper, lower rows respectively). The network response is seen to have an initial transient due to the default initial conditions of the RNN (eq. (20e, f)).

The third column of each of Figures 10(a-c) compares the steady-state orbital motion of the journal centre $\mathrm{J}_{1}$ relative to the bearing housing centre $\mathrm{B}_{1}$ within the clearance ( $y$ relative displacement vs $x$ relative displacement), it is seen that the predicted RNN orbits and the true ones in terms of orientation is reasonably good agreement. However, in term of size needs to be improved the training procedure.

Figures 11(a-c) provides the results for the RH-side SFD, as showed in Figure 1. In their first columns, it is again observed that the correlation between the $x, y$ forces within $\mathrm{d}_{\mathbf{f}_{\mathbf{v}_{2}}}(t)$ (solid lines) with the true ones within ${ }^{\mathrm{d}_{\mathbf{f}_{\mathbf{v}_{2}}}}(t)$ (dotted 
lines) is consistent to the ${ }^{d} \breve{f}_{\mathbf{v}_{1}}(t)$. Therefore, it is possible to obtain the SFD forces using the inversion of eq. (13). This inversion enables to obtain ${ }^{\mathrm{d}} \breve{\mathbf{f}}_{\mathbf{v}_{n}}(t)$ by using just the external vibration response and the modal parameters at each $\mathbf{B}_{n}$ terminal. Turning now to their second columns, where is observed that the initial transient phase of the RNN response signals in $\breve{\mathbf{v}}_{2}(t)$ takes considerably longer. This transient behaviour is due to the initial conditions described in eq. (20e, f). Another possible explanation for this is that the RNN mimics the mean position, which is determined by the equilibrium between the rotor weight at $\mathrm{J}_{2}$ (section 3.1) and the mean SFD force in the $y$-direction, which is itself a function of the vibration of $J_{2}$. This is also observed in the previous case (see Figure 10(b)) because both SFDs are unsupported.
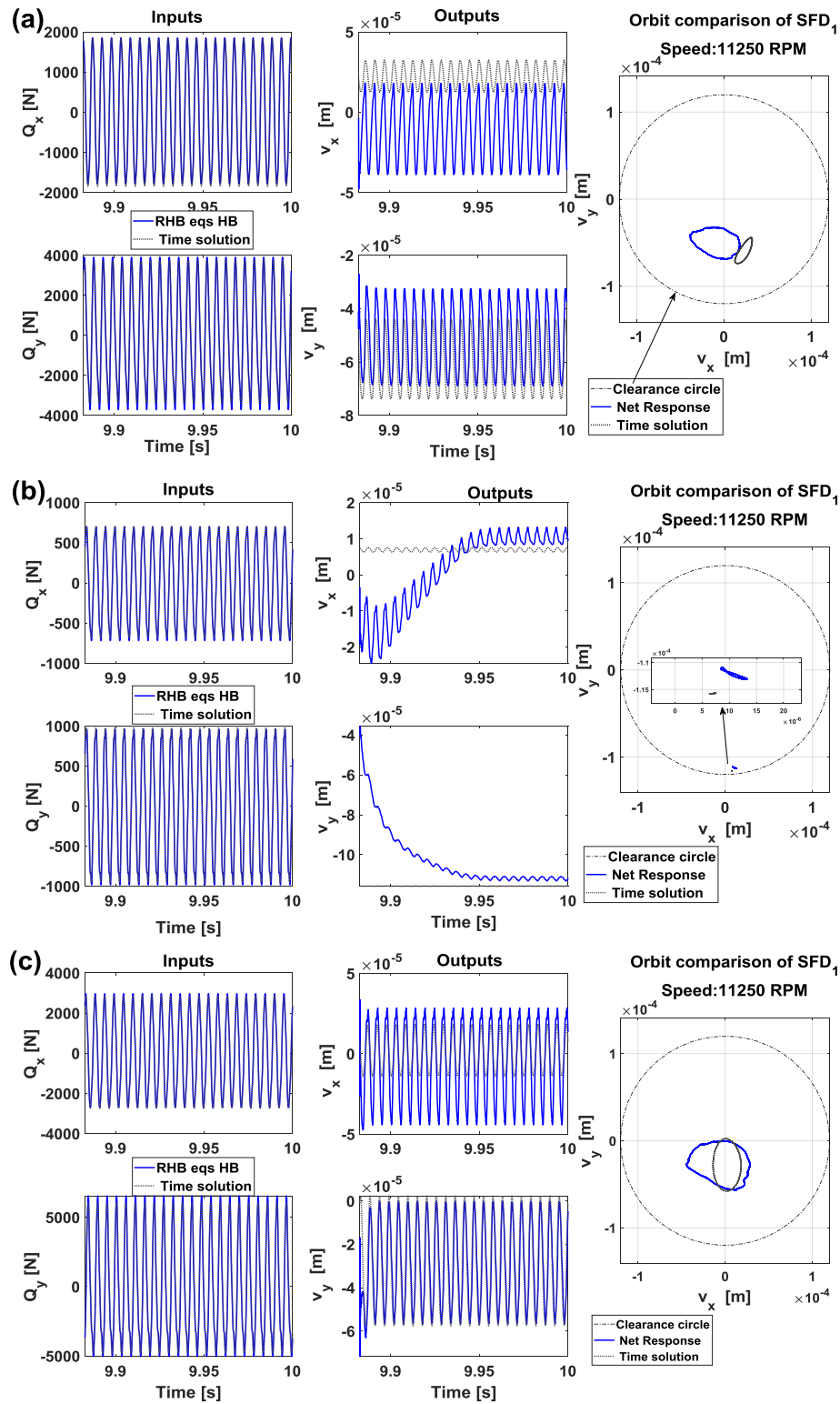

Figure 10. Comparison of the signals ${ }^{d} \breve{\mathbf{f}}_{\mathbf{v}_{1}}(t), \check{\mathbf{v}}_{1}(t)$ with the "true" signals ${ }^{d} \mathbf{f}_{\mathbf{v}_{1}}(t), \mathbf{v}_{1}(t)$ at the left-hand side SFD bearing (see figure 1) under the unbalance conditions of Table 1 and 11250 RPM: (a) Case 1, (b) Case 2, (c) Case 3.
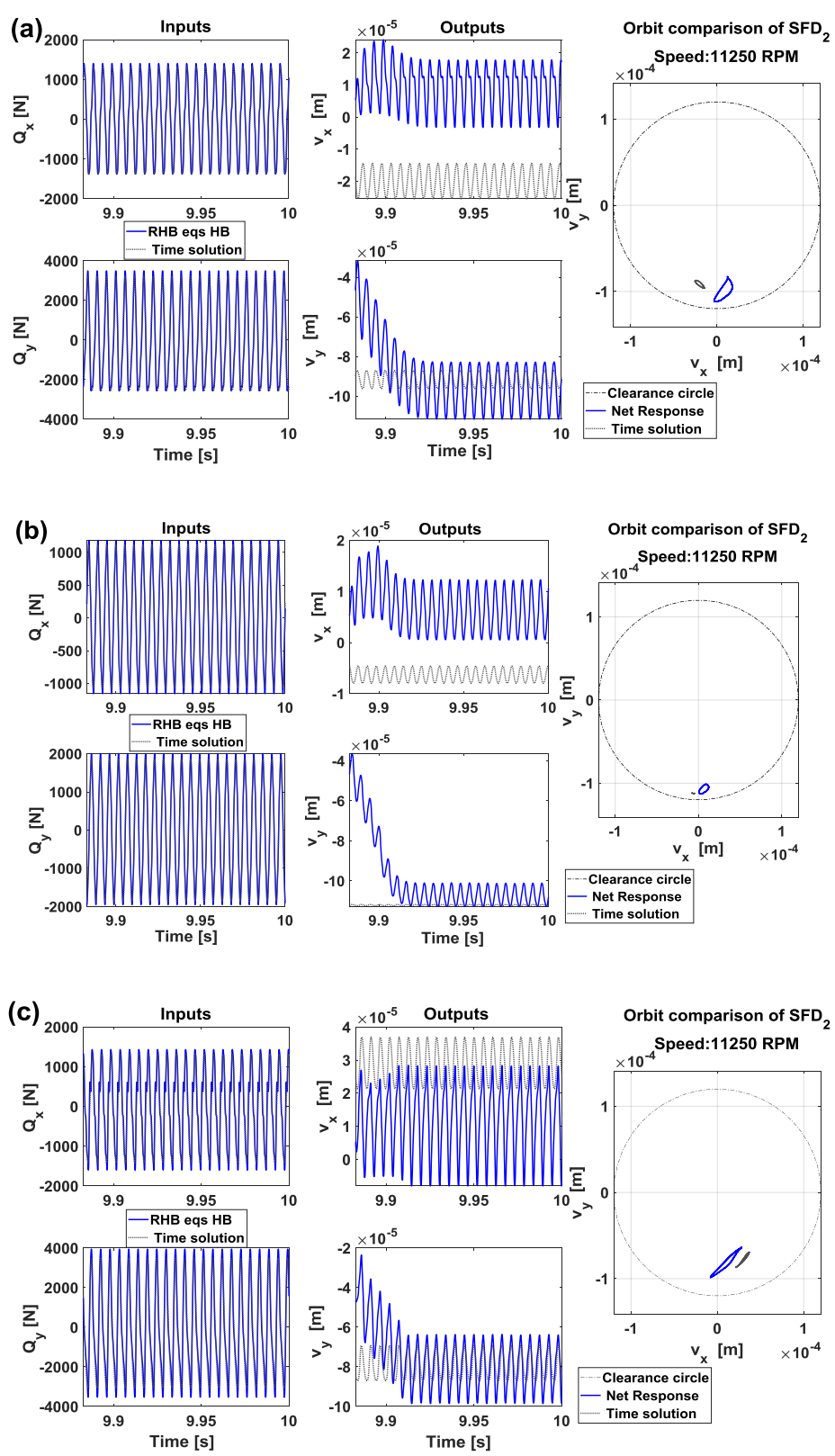

Figure 11. Comparison of the signals ${ }^{d} \breve{\mathbf{f}}_{\mathbf{v}_{2}}(t), \breve{\mathbf{v}}_{2}(t)$ with the "true" signals ${ }^{\mathrm{d}} \mathbf{f}_{\mathbf{v}_{2}}(t), \mathbf{v}_{2}(t)$ at the right-hand side SFD bearing (see figure 1) under the unbalance conditions of Table 1 and 11250 RPM: (a) Case 1, (b) Case 2, (c) Case 3.

In general, the following findings were attained when it was compared the time histories of $\check{\mathbf{v}}_{1,2}(t)$ and $\mathbf{v}_{1,2}(t)$ and considering different unbalance state conditions:

1. On average, the RNNs predictions were shown to have a positive correlation in correctly tracking the fundamental frequencies of the corresponding true signals.

2. The most disappointing observation to emerge from these RNN predictions is that there is a significant discrepancy in the amplitude of the time histories $\breve{\mathbf{v}}_{1,2}(t)$, especially on $x$ direction. 
3. Comparing the results presented in figures $10(a-c)$ and 11(a-c), one can see that the orbital plots have a reasonably good agreement with regards to mean position and orbital orientation when it is viewed within the clearance circle.

\subsection{STUDY CASE 2: RNN-PERFORMANCE OVER SEVERAL OPERATIONAL SPEEDS}

The previous section presented the evaluations between the RNN-predictions and the true ones at just one operational speed. From these comparisons, it was noted that the parameters mostly observed are: the orbit size and mean position when it is viewed within the clearance circle (orbital plots). Hence, the current section presents a study, which involves the network performance at 27 operational speeds $(8000,8250,8500,8750 \ldots, 14250$ and $14500 \mathrm{rpm})$ which cover the typical operating speed range of the HP rotor of an aero-engine. Therefore, the orbit size and mean position were calculated by the following procedure for each operational speed:

(i) Remove mean values of $\mathbf{v}_{1,2}(t)$ and $\breve{\mathbf{v}}_{1,2}(t)$ to obtain $\mathrm{d}_{\mathbf{v}, 2}(t)$ and $\breve{\mathbf{v}}_{1,2}(t)$. It is done to obtain the vibration amplitude peak to peak.

(ii) The mean values of $\overline{\mathbf{v}}_{1,2}$ and $\overline{\mathbf{v}}_{1,2}$ are also obtained from previous step. These values are obtained to estimate the Cartesian position of the orbit centre.

(iii) With reference to Figure 12, one can see that the orbit mean position can be expressed using polar coordinates, where $\vec{r}$ is a vector position and $\varphi$ its angle. Therefore, the mean values previously obtained were employed as follows.

$$
\begin{aligned}
& |\vec{r}|=\left.\sqrt{\left(\overline{\mathrm{v}}_{x_{1,2}}\right)^{2}+\left(\overline{\mathrm{v}}_{y_{1,2}}\right)^{2}}\right|_{\Omega_{k}, k=1 \ldots n_{\mathrm{o}}} \\
& \varphi=360^{\circ}-\left[\tan ^{-1}\left(\frac{\left.\overline{\mathrm{v}}_{y_{1,2}}\right)}{\overline{\mathrm{v}}_{x_{1,2}}}\right)\right] \times\left.\frac{180^{\circ}}{\pi}\right|_{\Omega_{k}, k=1 \ldots n_{\mathrm{o}}}
\end{aligned}
$$

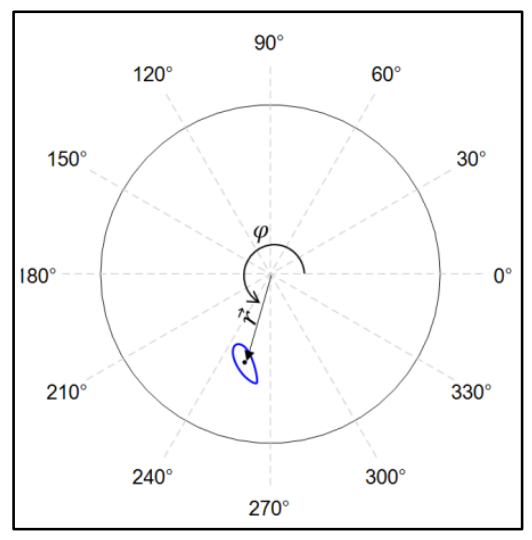

Figure 12. Example of a vector orbit position using polar coordinates. True $\mathrm{SFD}_{2}$ orbit plot obtained from unbalance case 1 at 10500 RPM.
The procedure described earlier has been done over 27 operational speeds to evaluate the two SFD inverse models based on artificial neural networks and considering three different unbalance state conditions as well. The results from the LH SFD 1 inverse model are summarized in Figure 13. The upper row in this figure presents comparison between the true values (dotted lines) ${ }^{\mathrm{d}} \mathbf{v}_{1}(t)$ and the RNN-predicted values (solid lines) $\mathrm{d}_{\mathbf{v}_{1}}(t)$., displacements are relative amplitudes peak-to-peak without the mean component. From these data, one can see that the amplitude from the network predictions are always bigger than the true amplitudes at the three unbalance cases. However, there is a clear trend in terms of tracking the dynamic behaviour, which is consistent over all considered unbalance cases.

The lower row from Figure 13, presents the results obtained from a comparison between the network predictions (solid lines) and the true ones (dotted lines), concerning the polar components of the vector position $\vec{r}$. These components are $|\vec{r}|$ (magnitude) and its $\varphi$ (angle). A significant positive correlation was noted in terms of magnitude $|\vec{r}|$ at the three unbalance state cases. Likewise, $\varphi$ is consistent over all speeds and unbalance cases, there is not a significant different between the predicted values and true values.

Figure 14 presents the corresponding results for the RH $\mathrm{SFD}_{2}$. It is apparent that the relative displacement amplitudes from ${ }^{\mathrm{d}} \mathbf{v}_{2}(t)$ and ${ }^{\mathrm{d}} \breve{\mathbf{v}}_{2}(t)$ were consistent with the results

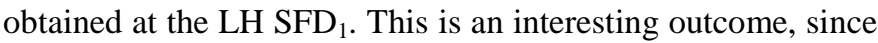
both SFD networks have a different architecture and training data as described in Section 4. Other remarkable results were obtained in the lower row, where again a significant encouraging correlation is observed in terms of magnitude $(|\vec{r}|)$ and angle $(\varphi)$. These results add to a growing body of evidence that proves the applicability of artificial neural network to model highly nonlinear bearing such as unsupported SFD bearings.
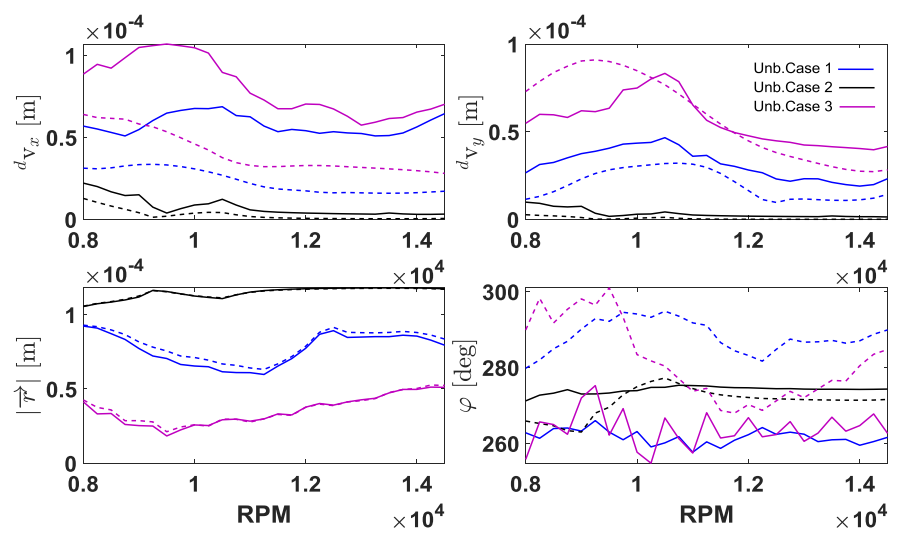

Figure 13. $\mathrm{SFD}_{1} \mathrm{RNN}$-performance over 27 operational speeds and considering three different unbalance state conditions. True response (dotted lines) and Network response (solid lines). 

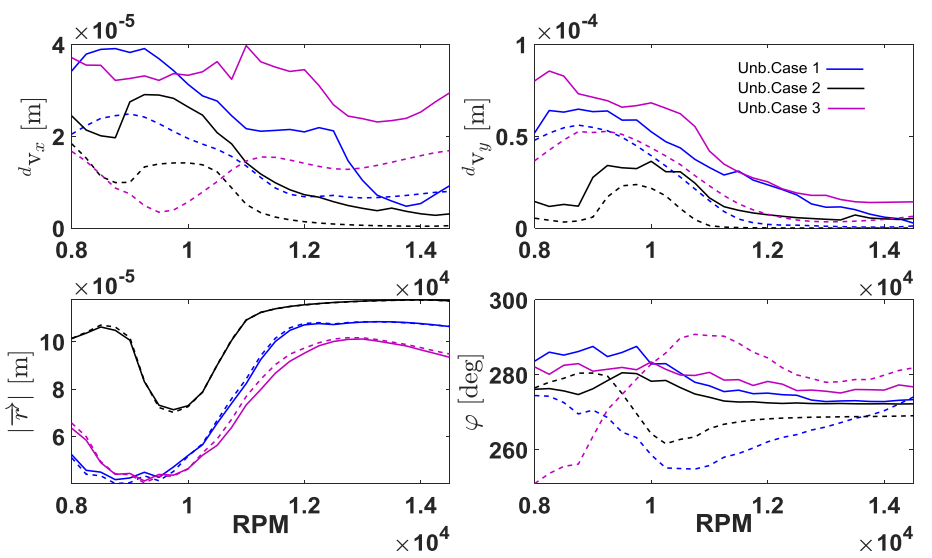

$10^{4}$

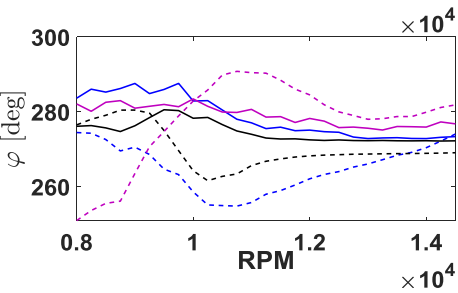

Figure 14. $\mathrm{SFD}_{2}$ RNN-performance over 27 operational speeds and considering three different unbalance state conditions. True response (dotted lines) and Network response (solid lines).

\section{CONCLUSIONS}

The aim of the present research was to examine the effectiveness of the inverse SFD model based on RNN when it is applied to a rotor-casing system wherein the rotor runs on two unsprung SFD bearings. One main motivation of this work was the requirement for a reliable substitute for internal instrumentation for applications where the rotor is not reachable under operating conditions and any explicit linear connections between rotor and casing, which is the case of the current SFD rotodynamic system. Therefore, this is the first study that has evaluated the effectiveness of two suitably trained SFD inverse models based on the NARX scheme which considers the current value of the outputs as a function of the past values of both the inputs and the outputs itself ("System memory"). These RNN models were shown to be able of identifying the inverse models of two unsprung SFDs via two numerical study cases. The results from these study cases show that the trained RNNs can be employed as an estimator for the SFD relative displacements. It also noted from these results that the RNNs can correctly track the fundamental frequency of the inputs and had reasonably good agreement with regards to mean position and orbital orientation, within the clearance circle. However, there is a significant disagreement in the orbit size which needs to be improved in order to be applied to a nonlinear inverse rotorbearing problem.

The results also indicate that both trained SFD networks have reasonable predictions for three different unbalance cases and 27 operational speeds. This finding encourages further research into the use of these networks as virtual internal sensors for the identification of the unbalance response from external (casing-mounted) sensors in a rotodynamic system that has any linear connections between rotor and casing. Another example of such a system is a turbocharger on floating ring bearings (FRBs) and a similar approach that uses suitably trained inverse RNN models for FRBs [32] can be explored.

\section{ACKNOWLEDGMENTS}

The authors would like to acknowledge the support from DGAPA-UNAM through its postdoctoral fellowship program named as "Programa de Becas Postdoctorales en la UNAM" and the Mexican Researcher's National System (SNICONACYT). Likewise, to the projects PAPIIT IN117717 and PAPIME PE100518. The authors are also grateful to The Higher Committee for Education Development in Iraq for financially supporting the current research.

\section{NOMENCLATURE}

\begin{tabular}{|c|c|}
\hline $\mathrm{d}()$ & $\begin{array}{l}\text { Denotes the exclusion of the mean } \\
\text { component from the quantity }()\end{array}$ \\
\hline$\overbrace{(\tau)}^{(\tau)}$ & $\begin{array}{l}\text { Denotes the network-normalised version of } \\
\text { the variable ( ) }\end{array}$ \\
\hline $\mathrm{B}_{n}$ & Bearing Housing terminal \\
\hline $\mathbf{f}_{\mathrm{u}}$ & Vector of unbalance forces. \\
\hline & Vector of SFD forces $(\mathrm{N})$ \\
\hline$\overline{\mathbf{f}}_{\mathbf{v}}, \mathbf{f}_{\mathbf{v}_{\mathbf{c}}}^{(\mathrm{s})}, \mathbf{f}_{\mathbf{v}_{\mathbf{s}}}^{(\mathrm{s})}$ & Fourier coefficients of SFD forces. \\
\hline g & Gyroscopic moment vector, Eq. 6 \\
\hline$G$ & $\begin{array}{l}\text { Total number of gyroscopic locations, Sec. } \\
3.1\end{array}$ \\
\hline $\mathbf{H}_{M}$, & $\begin{array}{l}\text { Modal matrices whose columns are } \\
\text { respectively the eigenvectors evaluated in } x, y\end{array}$ \\
\hline $\mathbf{H}_{\mathbf{u}}, \mathbf{H}_{\mathrm{g}}$ & $\begin{array}{l}\text { directions at the locations of the entries } \\
\text { in } \mathbf{x}_{\mathbf{p}_{i}}, \mathbf{f}_{\mathbf{u}}, \mathbf{g} \text {. Sec.3.1 }\end{array}$ \\
\hline $\mathbf{H}_{\mathbf{J}_{n}}, \mathbf{H}_{\mathbf{B}_{n}}$ & $\begin{array}{l}\text { Modal matrices whose columns are } \\
\text { respectively the eigenvectors evaluated in } x, y \\
\text { directions at terminals } \mathrm{J}_{n}, \mathrm{~B}_{n} \text { respectively. } \\
\text { Sec. } 3.1\end{array}$ \\
\hline $\mathbf{H}_{\mathbf{v}_{n}}$ & $=\mathbf{H}_{\mathbf{J}_{n}}-\mathbf{H}_{\mathbf{B}_{n}}$, Sec. 3.1 \\
\hline I & Identity matrix \\
\hline$I_{p}$ & $\begin{array}{l}\text { polar moment of inertia at location } p \\
(p=1, \ldots, G) \text {, sec. } 3.1\end{array}$ \\
\hline $\mathrm{J}_{n}$ & Journal Bearing terminal. \\
\hline$n$ & Number of SFD bearing \\
\hline$n_{o}$ & Number of operational speeds \\
\hline $\mathbf{M}_{\mathbf{p}_{i} \mathbf{v}}(\Omega)$ & Mobility matrix evaluated at $\Omega$. Sec. 3.2 \\
\hline $\mathrm{P}_{i}$ & Number of sensor location \\
\hline $\mathbf{q}$ & Vector of modal coordinates. \\
\hline$U_{k}, \phi_{\mathrm{k}}$ & $\begin{array}{l}\text { Unbalance forces and its angular position } \\
\text { with respect to reference phasor marker at } \\
\text { disk no. } k \text {. }\end{array}$ \\
\hline$\vec{r}, \varphi$ & Polar coordinates \\
\hline$r$ & Counter for modes \\
\hline$R$ & Total number of modes considered \\
\hline$S$ & $\begin{array}{l}\text { Positive integer, equal to the order of a } \\
\text { harmonic in RHBM equations }\end{array}$ \\
\hline$S^{(j)}$ & Number of neurons in the layer $\mathrm{j}^{\text {th }}$ \\
\hline$t$ & Time $(s)$ \\
\hline$t_{k}$ & Discrete time (s) \\
\hline $\mathbf{v}_{\mathbf{n}}$ & $\begin{array}{l}\text { Vector of the instantaneous Cartesian }(x, y) \\
\text { displacements of } \mathrm{J}_{n} \text { relative to } \mathrm{B}_{n},\end{array}$ \\
\hline $\mathbf{w}_{1,2}$ & $\begin{array}{l}\text { Vector of the equivalent static loads at } \mathrm{J}_{1,2} \text { in } \\
\text { the } x, y \text { directions. }(\mathrm{N})\end{array}$ \\
\hline
\end{tabular}




$\begin{array}{cl}\mathbf{x}_{\mathbf{p}} & \begin{array}{l}\text { Vector of displacements at Cartesian sensor } \\ \text { locations. (m) } \\ \text { Vector of velocity readings at Cartesian } \\ \text { sensor locations. (m/s) }\end{array} \\ \dot{\mathbf{x}}_{\mathbf{p}} & \begin{array}{l}\text { Fourier coefficients of displacements at } \\ \text { Cartesian sensor locations. }\end{array} \\ \overline{\mathbf{x}}, \mathbf{x}_{\mathbf{p}_{\mathbf{c}}}^{(\mathrm{s})}, \mathbf{x}_{\mathbf{p}_{\mathbf{s}}}^{(\mathrm{s})} & \begin{array}{l}\text { Fourier coefficients of velocities at Cartesian } \\ \text { sensor locations. }\end{array} \\ \mathbf{z}_{\mathbf{p}_{i}}^{(s)}, \mathbf{z}_{\mathbf{p}_{i S}}^{(s)} & \begin{array}{l}\text { Angle of location of the stationary phase } \\ \text { probe (deg). }\end{array} \\ \gamma & \text { The sampling time resolution } \\ \Delta & \text { The diagonal matrix of squares of natural } \\ \boldsymbol{\Lambda} & \text { frequencies. } \\ \boldsymbol{\Psi}_{\mathbf{J}_{n}}^{(r)}, \boldsymbol{\Psi}_{\mathbf{B}_{n}}^{(r)} & \begin{array}{l}\text { Mass-normalized eigenvector defining the x } \\ \text { and y displacements of the squeeze-film } \\ \text { terminals J, B respectively in mode no. } r .\end{array} \\ \boldsymbol{\Psi}_{\mathbf{v}_{n}}^{(r)} & =\boldsymbol{\Psi}_{\mathbf{J}_{n}}^{(r)}-\boldsymbol{\Psi}_{\mathbf{B}_{n}}^{(r)} \\ \Omega & \text { Rotational speed (rad/s) } \\ \omega_{r} & \text { Natural frequency (rad/s) in mode no. } r\end{array}$

\section{REFERENCES}

[1] Cooper, S., 1963, "Preliminary Investigation of Oil Films for the Control of Vibration," Lubr. Wear Conv., pp. 305-315.

[2] Adiletta, G., 2002, "The Squeeze Film Damper over Four Decades of Investigations. Part I: Characteristics and Operating Features," Shock Vib. Dig., 34, pp. 3-26.

[3] Zeidan, F. Y., San Andres, L., and Vance, J. M., 1996, "Design and Application of Squeeze Film Dampers in Rotating Machinery," Proceedings of the 25th Turbomachinery Symposium, pp. 169-188.

[4] San Andrés, L., and Jeung, S.-H., 2014, "Experimental Performance of an Open Ends, Centrally Grooved, Squeeze Film Damper Operating With Large Amplitude Orbital Motions," J. Eng. Gas Turbines Power, 137(3), pp. 3250832509.

[5] Torres Cedillo, S. G., Al-Ghazal, G. G., Bonello, P., and Cortés Pérez, J., 2019, "Improved Non-Invasive Inverse Problem Method for the Balancing of Nonlinear Squeeze-Film Damped Rotordynamic Systems,” Mech. Syst. Signal Process., 117, pp. 569-593.

[6] Holmes, R., and Dogan, M., 1982, "Investigation of a Rotor Bearing Assembly Incorporating a Squeeze-Film Damper Bearing," J. Mech. Eng. Sci., 24(3), pp. 129-137.

[7] Torres Cedillo, S. G., and Bonello, P., 2016, "Empirical Identification of the Inverse Model of a Squeeze-Film Damper Bearing Using Neural Networks and Its Application to a Nonlinear Inverse Problem,” J. Vib. Control., 24(2), pp. 357378

[8] Chen, Z., Jiao, Y., Xia, S., Huang, W., and Zhang, Z., 2002 , "An Efficient Calculation Method of Nonlinear Fluid Film Forces in Journal Bearing,” Tribol. Trans., 45(3), pp. 324-329.

[9] Groves, K. H., Bonello, P., and Hai, P. M., 2012, "Efficient Dynamic Analysis of a Whole Aeroengine Using Identified Nonlinear Bearing Models," Proc. Inst. Mech. Eng. Part C J. Mech. Eng. Sci., 226(1), pp. 66-81.

[10] Groves, K. H., and Bonello, P., 2010, "Improved Identification of Squeeze-Film Damper Models for Aeroengine Vibration Analysis," Tribol. Int., 43(9), pp. 1639-1649.

[11] Rodrigues, F. A., Thouverez, F., Gibert, C., and Jezequel, L., 2003, "Chebyshev Polynomials Fits for Efficient Analysis of Finite Length Squeeze Film Damped Rotors," J. Eng. Gas Turbines Power, 125(1), pp. 175-183.

[12] Groves, K. H., and Bonello, P., 2013, "Empirical Identification of Squeeze-Film Damper Bearings Using Neural Networks," Mech. Syst. Signal Process., 35(1-2), pp. 307-323.

[13] McCormick, A. C., and Nandi, A. K., 1997, "Real-Time
Classification of Rotating Shaft Loading Conditions Using Artificial Neural Networks," Neural Networks, IEEE Trans., 8(3), pp. 748-757.

[14] Reddy, M. C. S., and Sekhar, A. S., 2013, "Application of Artificial Neural Networks for Identification of Unbalance and Looseness in Rotor Bearing System,” Int. J. Appl. Sci. Eng., 11(1), pp. 69-84.

[15] Walker, R. B., Vayanat, R., Perinpanayagam, S., and Jennions, I. K., 2014, "Unbalance Localization through Machine Nonlinearities Using an Artificial Neural Network Approach," Mech. Mach. Theory, 75(0), pp. 54-66.

[16] Dicken, V., Maaß, P., Menz, I., Niebsch, J., and Ramlau, R., 2005, "Nonlinear Inverse Unbalance Reconstruction in Rotor Dynamics," Inverse Probl. Sci. Eng., 13(5), pp. 507-543.

[17] Torres Cedillo, S. G., and Bonello, P., 2014, "Unbalance Identification and Balancing of Nonlinear Rotodynamic Systems," Vol. 7A Struct. Dyn., pp. V07AT31A004.

[18] Torres Cedillo, S. G., and Bonello, P., 2016, “An Equivalent Unbalance Identification Method for the Balancing of Nonlinear Squeeze-Film Damped Rotordynamic Systems," J. Sound Vib., 360, pp. 53-73.

[19] Al-Ghazal, G. G., Bonello, P. and Torres Cedillo, S. G., 2018, "Improved Empirical Identification of the Inverse Model of a Squeeze-Film Damper Bearing Based on a Recurrent Neural Network," Vol. 7A Struct. Dyn., pp. V07AT33A016.

[20] Ramlau, R., Dicken, V., Maß, P., Streller, C., and Rienäcker, A., 2006, "Inverse Imbalance Reconstruction in Rotordynamics," ZAMM - J. Appl. Math. Mech. / Zeitschrift für Angew. Math. und Mech., 86(5), pp. 385-399.

[21] Bonello, P., and Minh Hai, P., 2009, "A Receptance Harmonic Balance Technique for the Computation of the Vibration of a Whole Aero-Engine Model with Nonlinear Bearings," J. Sound Vib., 324(1-2), pp. 221-242.

[22] Dede, M. M., Dogan, M., and Holmes, R., 1985, "The Damping Capacity of a Sealed Squeeze Film Bearing," J. Tribol., 107(3), pp. 411-418.

[23] Hai, P. M., and Bonello, P., 2008, “An Impulsive Receptance Technique for the Time Domain Computation of the Vibration of a Whole Aero-Engine Model with Nonlinear Bearings," J. Sound Vib., 318(3), pp. 592-605.

[24] Bonello, P., and Hai, P. M., 2009, "Computational Studies of the Unbalance Response of a Whole Aero-Engine Model With Squeeze-Film Bearings,” J. Eng. Gas Turbines Power, 132(3), p. 32504.

[25] Reymond, M., 2005, "MSC . Nastran realase guide".

[26] Adams, M. L., 1980, "Non-Linear Dynamics of Flexible MultiBearing Rotors," J. Sound Vib., 71(1), pp. 129-144.

[27] Bonello, P., Brennan, M. J., and Holmes, R., 2003, “An Investigation into the Non-Linear Dynamics of an Unbalanced Flexible Rotor Running in an Unsupported Squeeze-Film Damper Bearing,” Proc. Inst. Mech. Eng. Part C J. Mech. Eng. Sci., 217(8), pp. 955-971.

[28] Haykin, S., 1999, Neural Networks: A Comprehensive Foundation, Prentice Hall.

[29] Beale, M. H., Hagan, M. T., and Demuth, H. B., 2010, "Neural Network Toolbox 7," User's Guide. MathWorks.

[30] Shampine, L., and Reichelt, M., 1997, "The MATLAB ODE Suite,” SIAM J. Sci. Comput., 18(1), pp. 1-22.

[31] Torres Cedillo, S. G., Bonello, P., Al-Ghazal, G. G.,Cortés Pérez, J. and Reyes Solis A., 2018, "Effectiveness testing of an inverse method for balancing nonlinear rotordynamic systems," Vol. 7A Struct. Dyn., p. V07AT33A005.

[32] Bonello, P., 2009, "Transient Modal Analysis of the Non-Linear Dynamics of a Turbocharger on Floating Ring Bearings," Proc. Inst. Mech. Eng. Part J J. Eng. Tribol., 223(1), pp. 79-93. 